\title{
A New Approach for Estimating Parameters in PV Cell Models based on Odd Polynomial Regression
}

\author{
Ahmed Abdolkhalig*, Ashraf Mohamed, Fatihe Abusief \\ Department of Electrical Engineering, The University of Tobruk, 4004, Tobruk, Butnan, Libya.
}

\begin{abstract}
How to cite this paper: Ahmed Abdolkhalig, Ashraf Mohamed, Fatihe Abusief. (2022) A New Approach for Estimating Parameters in PV Cell Models based on Odd Polynomial Regression. Journal of Electrical Power \& Energy Systems, 6(1), 17-23.

DOI: $10.26855 /$ jepes.2022.01.002
\end{abstract}

Received: December 2, 2021

Accepted: December 27, 2021

Published: January 21, 2022

*Corresponding author: Ahmed Abdolkhalig, Department of Electrical Engineering, The University of Tobruk, 4004, Tobruk, Butnan, Libya.

Email: ahmed@tu.edu.ly

\begin{abstract}
This paper proposes a simple approach for estimating three of the parameter values of photovoltaic cell that can be modelled as a single-diode equivalent circuit model. The proposed method relies on the assumption that in the silicon-based single-diode equivalent circuit, the shunt resistance has a very high value and thus, its current effect can be neglected. This negligence can enable us to easily convert any photo-illuminated current based model from a logarithmic regression model to a simple odd polynomial regression model. The resulted polynomial regression model can enable us to simply estimate three parameters of the intrinsic parameters of single-diode equivalent circuit model and also, it can be applied to the characterization of any typical photovoltaic cell at varied temperatures. Root mean square error would be considered as an accuracy criterion to evaluate the estimation performance errors when the degree of the polynomial is iterated. The method is both analytically and the soft computing are covered and finally, results are discussed.
\end{abstract}

\section{Keywords}

Photovoltaic cell model, logarithmic model, polynomial model, regression, parameters estimation

\section{Introduction}

The major problem related to the photovoltaic (PV) cell model building is the computational challenge of parameters estimation related to internal properties of the cell such as resistances $\left(R_{S}\right)$, saturation current $\left(I_{o}\right)$ and the ideality factor $(\eta)$ of the diode junction.

Recently, there are many research works that used different regression methods for estimating other different parameters. Most of these research works have focused only on the estimation of PV output power based on several factors of climate conditions such as irradiance, wind; dust and temperature [1-5]. Moreover, due to the significant effect of parameters related to internal properties (i.e. $R_{s}, I_{o}$ and $\eta$ ) on PV output power, many research studies [2, 3, 5-7] have been carried out for accurate estimation of these parameters.

The methods that were suggested in these research works are mainly rely on deferent regression techniques such as, linear and nonlinear regressions, multiple-linear regressions, Gaussian process regression and parallel branch regression formulation. Also, proposed methods in [8-11] are quite extensive because, they do not simplify the PV equivalent circuit model and most of these methods can find parameter values by solving a system of non-linear equations. 
There are many techniques for obtaining the model of illuminated PV cells. One of those techniques is to use dark models that are using the Shockley diode as in equation 1, to find the I-V relation of the PV cell in zero-illumination condition.

$$
I_{D}=I_{\circ}\left(e^{V_{D} / \eta V_{T}}-1\right)
$$

where $V_{T}$ is thermal voltage and equal to $\frac{k T}{q}, q$ is the charge of an electron, $k$ is the Boltzmann constant, $T$ is the temperature in ${ }^{\circ} \mathrm{K}, I_{o}$ is the inverse saturation current of the diode and $n$ is the ideality factor which is measure of how closely a diode follows the ideal diode characteristic.

In illumination conditions, a solar PV cell may represented by a single-diode equivalent circuit model as shown in Figure 1.

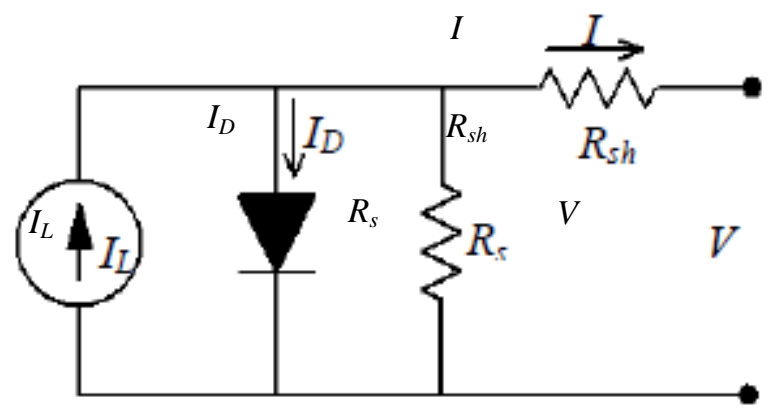

Figure 1. A single-diode equivalent circuit with series and parallel resistance.

This equivalent circuit also known as five parameters model $\left(I_{L}, I_{o}, R_{S}, R_{S h}, \eta\right)$, where $I_{L}$ is photo-generated current, $R_{s}$ and $R_{s h}$ are resistors that modeling the voltage drop $\left(V_{s}\right)$ and the current loss $\left(I_{s h}\right)$, respectively. The photo-illuminated current and current loss offset the I-V curve modeled as in (1) from origin as follows:

$$
I=I_{L}-I_{D}-I_{\text {sh }}
$$

Substitute values of $I_{D}$ and $I_{s h}$ in (2), yields

$$
I=I_{L}-I_{\circ}\left(e^{V_{D} / \eta V_{T}}-1\right)-\frac{V-R_{S} I}{R_{S h}}
$$

The novelty in this paper is the equivalent circuit model is simplified by transforming it from a logistic nonlinear regression model to a simple odd polynomial regression. The method can be applied to any single-diode model incorporating as in (3). The method makes use of a full-range of available I-V curves. The accuracy is verified by recovering known parameter values from calculated data points and compared to measured data points.

The remaining of this paper is structured as follows: Section 2 presents the steps performed to transform a logarithmic model to a simple odd polynomial model for estimating three parameters for the single-diode model outlined in Figure 1. In Section 3, the results and verification are presented. The effects of polynomial degree and added white noise on model estimation errors are discussed in this section. Finally, Section 4 presents the conclusions.

\section{Method}

The solar PV model relationship can be best described in zero-illumination condition by the exponential model as in (4), which is resulted after ignoring the photo-illuminated current $\left(I_{L}\right)$ appeared in (3).

$$
I=I_{\circ}\left(e^{V_{D} / \eta V_{T}}-1\right)-\frac{V-R_{S} I}{R_{S h}}
$$

In the manufacturing of silicon-based diodes that are commonly used in energy applications, the shunt resistance 
$\left(R_{S h}\right)$ has a very high value. This can lead to make the term $V-R_{S} I / R_{S h}$ to have a very small value and thus, it can be neglected in (4). The single-diode equivalent circuit model can be reduced to an approximated model represented as in (5). This equation can be rearranged to represent the voltage as the dependent variable and the current as an independent variable as in (6).

$$
\begin{gathered}
I=I_{\circ}\left(e^{V-R_{s} I / \eta V_{T}}-1\right) \\
V=\eta V_{T} \ln \left(I_{\circ}\right)+R_{S} I+\eta V_{T} \ln (I)
\end{gathered}
$$

Apply Taylor series properties for logarithmic functions to (6), then it can be rewritten in a polynomial form as follows:

$$
\begin{aligned}
& V=-\eta V_{T} \ln \left(I_{\circ}\right)+R_{S} I+2 \eta V_{T}\left(\frac{I-1}{I+1}\right)+\ldots \\
& \frac{2}{3} \eta V_{T}\left(\frac{I-1}{I+1}\right)^{3}+\frac{2}{5} \eta V_{T}\left(\frac{I-1}{I+1}\right)^{5}+\ldots \\
& \frac{2}{n} \eta V_{T}\left(\frac{I-1}{I+1}\right)^{n}
\end{aligned}
$$

This is an equivalent to $n^{\text {th }}$ degree odd polynomial in the form:

$$
y=a_{0}+a_{1} x+a_{3} x^{3}+a_{5} x^{5} \ldots+a_{n} x^{n}
$$

The unknown coefficients $\left(a_{0}, a_{1} \rightarrow a_{\mathrm{n}}\right.$ ) can be estimated by doing polynomial regression fit. While polynomials with some higher degrees can be useful attempts to describe data points as fully as possible, incorporating higher degree terms may cause a numerical overflow and thus, a trade-off between the model degree size and its accuracy should be made. For this purpose, Monte-Carlo simulation runs $[12,13]$ will be performed to enable assessing the performance under several degrees of polynomial. This will assure that the required root mean square error (RMSE) accuracy is achieved for lower degree. The three parameters found in (5) (i.e. $R_{s}, I_{o}$ and $\eta$ ) can be calculated only from the first three coefficients (i.e. $a_{0}, a_{1}$ and $a_{3}$ ) of the polynomial as follows:

$$
\left.\begin{array}{l}
\eta=\frac{1.5 a_{3}}{V_{T}} \\
R_{s}=a_{1} \\
I_{\circ}=\log \left(\frac{-a_{0}}{n V_{T}}\right)
\end{array}\right\}
$$

\section{Computational Results and Verification}

The accuracy of the proposed method can be verified by computing simulated I-V curves at various temperatures $\left(0^{\circ} \mathrm{C}, 25^{\circ} \mathrm{C}, 50^{\circ} \mathrm{C}\right.$, and $\left.75^{\circ} \mathrm{C}\right)$ for (3) and then, applying the step-by-step procedure from (5) to (9) to extract the three parameters found in the single-diode equivalent circuit model. Monte Carlo simulation is used to model uncertainty due to noise added to the data points that are extracted from simulated I-V curves.

The concept of signal to noise ratio (SNR) that compares level of noise in measured signals is used in this paper (expressed in decibels) to ensure the best model noise functionality.

\subsection{Polynomial degree effect}

To evaluate the effect of the degree of the polynomial on the three parameter estimation errors, the noise is assumed zero and RMSE is calculated to measure of how the data is concentrated around the proposed model fit. The degree of the polynomial has been assigned an arbitrary value of 50 degrees. Because of equation 7 is an odd degree polynomial, the simulation should be executed for 25 iterations (i.e. $n / 2$ ) and at each time, polynomial degree and 
the corresponding RMSE should be recorded.

The impact of increasing the degree of the polynomial on the RMSE is shown in Figure 2. The figure shows that while the degree is increasing to a value of 25 degrees, the RMSE decreases rapidly to a very small value. At 25 degrees and afterward, values of RMSE can be assumed to have a zero value.

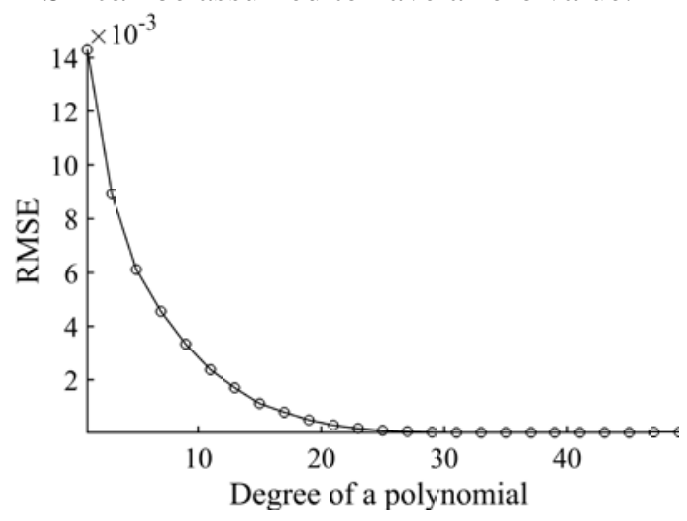

Figure 2. RMSE versus $n$.

It is more advantageous to evaluate the effect of polynomial degree directly on the extracted three parameters $\left(I_{o}\right.$, $R_{s}$ and $\eta$ ). Here, instead of using RMSE, \% percentage error is used as performance metric to measure the difference between exact and extracted values of parameters. As shown in Figure 3, the maximum \% error is recorded at one degree for the parameter $I_{o}$ which was about $15 \times 10^{3} \%$, while it was recorded at $250 \%$ and $50 \%$ for $R_{s}$ and $\eta$, respectively.
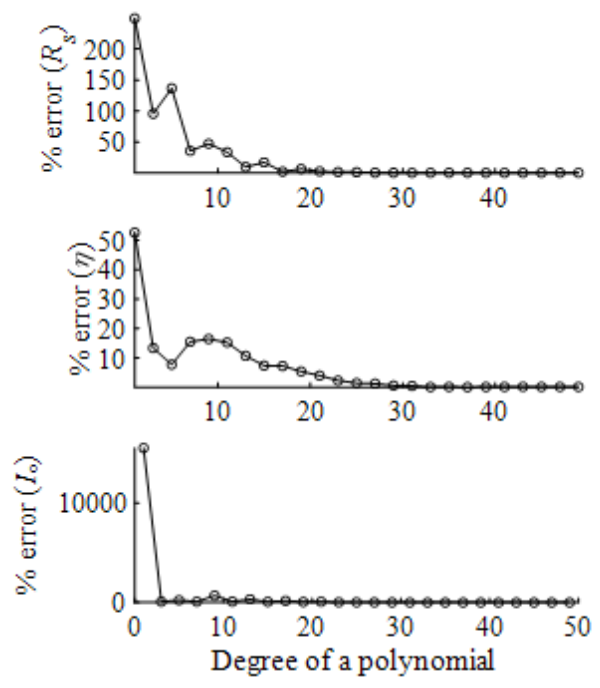

Figure 3. \% error of parameters versus $n$.

These \% errors have decayed rapidly to steady-state values of almost zeros and the parameter $I_{o}$ was the faster one (gets minimum at 3 degrees). Table 1 summarizes the results. From the table, it can be revealed that \% error for $I_{o}$ is decreased from $15 \times 10^{3} \%$ to $\approx 0 \%$ and from $250 \%$ to $\approx 0 \%$ and from $50 \%$ to $\approx 3 \%$ for $R_{\mathrm{s}}$ and $\eta$, respectively.

Table 1. Maximum and Minimum of \% Error

\begin{tabular}{cccc}
\hline Parameter & $I_{o}$ & $R_{s}$ & $\eta$ \\
\hline Max \% error [@ $n=1]$ & $15 \times 10^{3}$ & 250 & 50 \\
Min \% error [@ $n=25]$ & $\approx 0$ & $\approx 0$ & $\approx 3$ \\
\hline
\end{tabular}




\subsection{Noise Effect}

In PV cell energy applications, noise can play a curial rule in the estimation of the PV model parameters. The error term which is generally considered to arise from a number of sources, such as measurement noise and modelling bad fit. The errors raised in the estimation can be enormous and thus, the robustness of the method against such errors needs to be verified. To do so, white Gaussian noise was added to mimic the effect of noise in the simulated data.

Here, Monte Carlo simulation was tuned for values from a maximum noise (SNR=10) to a minimum noise $(\mathrm{SNR}=100)$ at various temperatures $\left(T=0^{\circ} \mathrm{C}, 25^{\circ} \mathrm{C}, 50^{\circ} \mathrm{C}\right.$, and $\left.75^{\circ} \mathrm{C}\right)$. Again, at each simulation; the curve data of SNR versus RMSE was recorded. Figure 4 shows the noise effect is rapidly decreases at a knee-noise values (e.g. $\mathrm{SNR}=14$ at $\left.75^{\circ} \mathrm{C}\right)$ and it appears that RMSE value becomes very small $(\approx 0 \%)$ when $\mathrm{SNR} \approx 50 \mathrm{~dB}$.

There are other statistical functions that can be used to evaluate the performance of the proposed model under the effect of the noise such as sum squared error (SSE) and R-squared. Figure 5 shows four graphs representing the performance at $T=0^{\circ} \mathrm{C}, 25^{\circ} \mathrm{C}, 50^{\circ} \mathrm{C}$, and $75^{\circ} \mathrm{C}$. Almost, all graphs settle at a steady-state value at $\mathrm{SNR} \approx 30 \mathrm{~dB}$ and the model has better performance at $T=0^{\circ} \mathrm{C}$ whereas, the SSE value is smaller compared to all other graphs at other temperatures.

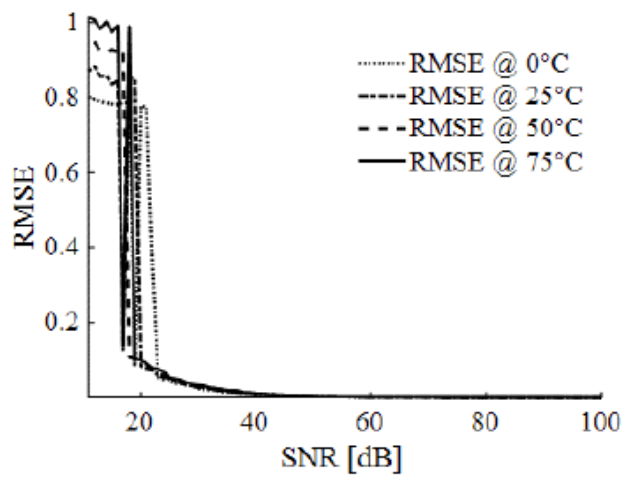

Figure 4. RMSE versus SNR.

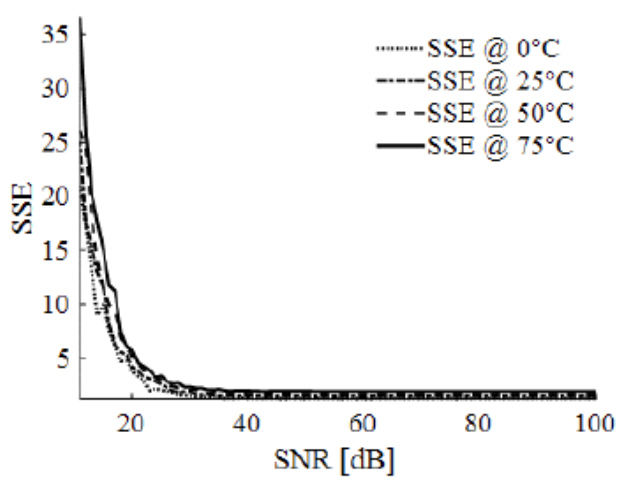

Figure 5. A plot of SSE versus SNR.

Graphs in Figure 6 show the test for R-squared versus SNR. All the graphs settle when SNR $\approx 30 \mathrm{~dB}$ (or over) at almost R-squared equals one which means superior model fitting.

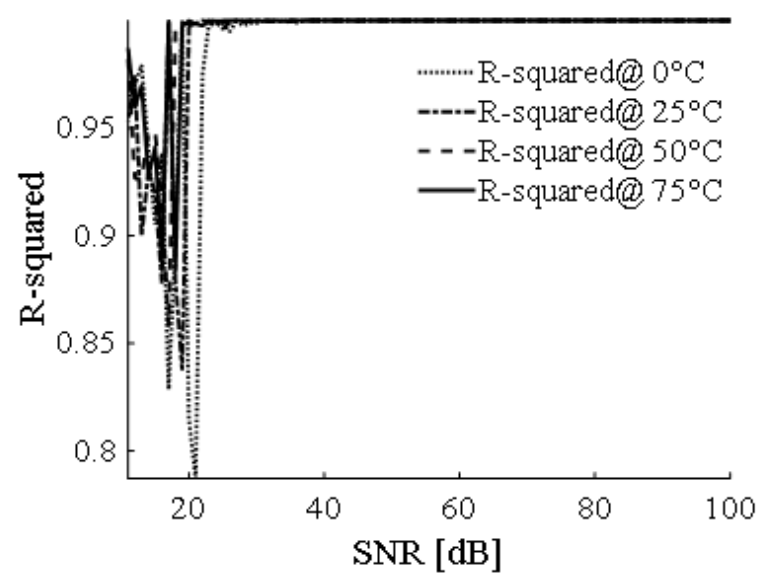

Figure 6. R-squared versus SNR.

The graph for $T=0{ }^{\circ} \mathrm{C}$ shows a maximum deviation value of $\mathrm{R}$-squared $(\approx 0.22)$ at $\mathrm{SNR} \approx 22 \mathrm{~dB}$. Table 2 gives the largest deviation values from one (Ideal case of R-squared) corresponding to SNR for all graphs. 
Table 2. Largest Deviation Values of R-Squared Values of R-squared

\begin{tabular}{ccccc}
\hline$T\left[{ }^{\circ} \mathrm{K}\right]$ & $0^{\circ} \mathrm{C}$ & $25^{\circ} \mathrm{C}$ & $50^{\circ} \mathrm{C}$ & $75^{\circ} \mathrm{C}$ \\
\hline SNR [dB] & 22 & 20 & 19 & 20 \\
R-squared & $\approx 0.22$ & $\approx 0.17$ & $\approx 0.165$ & $\approx 0.16$ \\
\hline
\end{tabular}

The performance of the proposed model can also be evaluated by using residual plots. Several residual plots can be used for assessing errors or correlations in the model such as default histogram plot and plotting residuals vs. fitted plot. The default histogram plot is considered the simplest residual plots that can show the residuals range and their occurrence. In Figure 7, the residuals appear to be randomly distributed around zero and the maximum recorded residual value was 0.01 when the temperature was $75^{\circ} \mathrm{C}$. In Figure 8, the histograms are plotted for residuals of 400 simulated data points. The plots look symmetric without an obvious problem. However, symmetric of these plots might be improved by increasing the data size (i.e. the number of measurements).

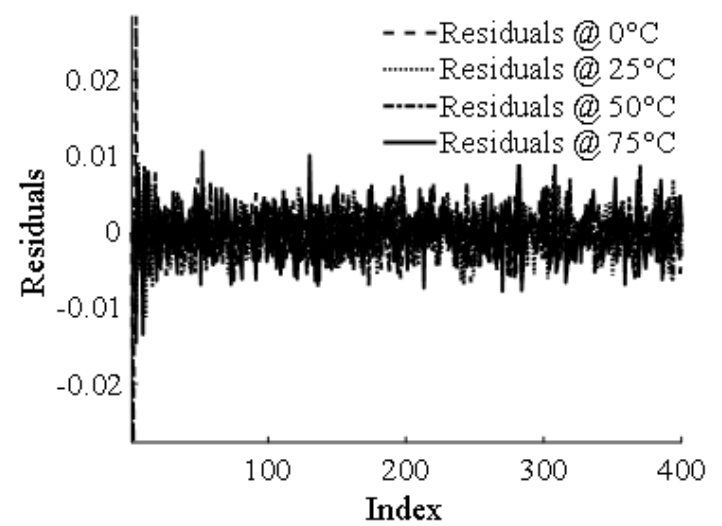

Figure 7. Residuals at various temperatures.
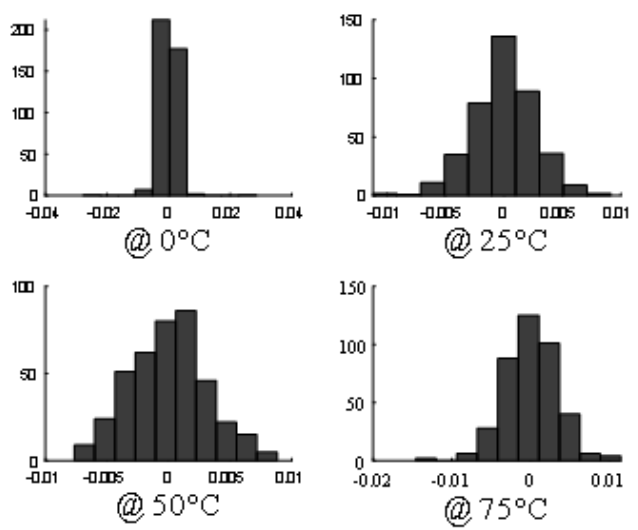

Figure 8. Histograms of residuals.

The second helpful way to plot the residuals is though plotting residuals vs. fitted as in Figure 9. It is clear from this figure that there are no outliers and fitted values being close to zero. Finally, Figure 10 demonstrates these results and shows both of a simulated I-V characteristic and the graphs of the proposed model given as in (5) for the three parameters $I_{o}, R_{s}$ and $\eta$ values that been estimated by the above approach.

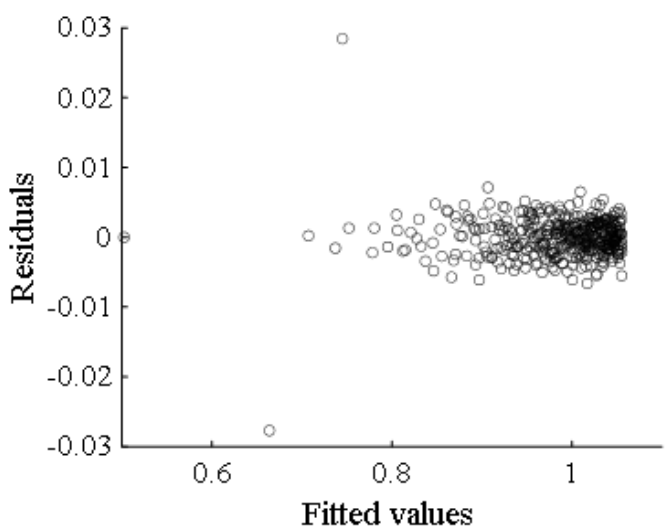

Figure 9. Residuals versus fitted values.

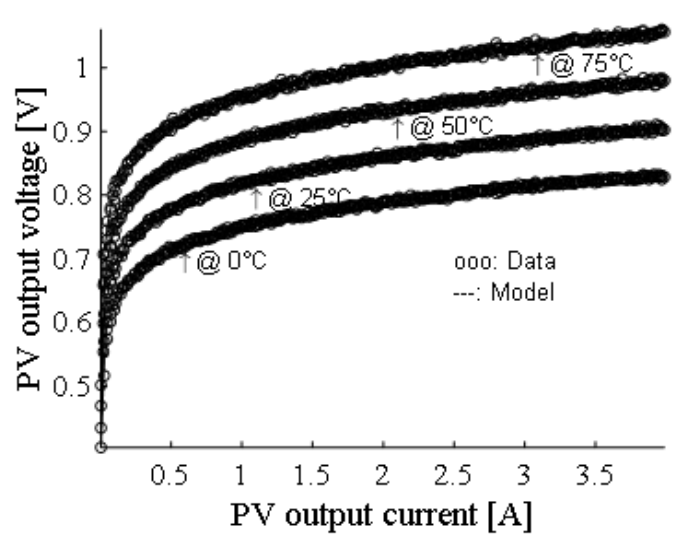

Figure 10. I-V curves for various temperatures.

\section{Conclusions}

A new approach for three parameters estimation in silicon-based single-diode equivalent circuit PV cell models with a large shunt resistance is presented in this paper. The approach required a prior transformation of the loga- 
rithmic single-diode equivalent circuit to the polynomial regression model. The results validated the performance of the approach, which further is improved by applies an iterative procedure to obtain the values of $I_{o}, R_{s}$, and $\eta$ for each I-V curve. The simplicity of the approach can make it easy to be implemented in solar energy applications. The proposed approach in this paper can be also further applied to more complicated PV models in the future.

\section{References}

[1] Almaktar, M., et al. (2015). Artificial neural network-based photovoltaic module temperature estimation for tropical climate of Malaysia and its impact on photovoltaic system energy yield. Progress in Photovoltaics: Research and Applications, 2015. 23(3): 302-318.

[2] De Leone, R., M. Pietrini, and A. Giovannelli. (2015). Photovoltaic energy production forecast using support vector regression. Neural Computing and Applications, 2015, 26(8): 1955-1962.

[3] Ibrahim, S., et al. (2012). Linear regression model in estimating solar radiation in Perlis. Energy Procedia, 2012, 18: 1402-1412.

[4] Rizwan, M., et al. (2014). Fuzzy logic based modeling and estimation of global solar energy using meteorological parameters. Energy, 2014, 70: 685-691.

[5] Wang, G., Y. Su, and L. Shu. (2016). One-day-ahead daily power forecasting of photovoltaic systems based on partial functional linear regression models. Renewable Energy, 2016, 96: 469-478.

[6] Kanwal, S., et al. (2018). Gaussian process regression based inertia emulation and reserve estimation for grid interfaced photovoltaic system. Renewable Energy, 2018, 126: 865-875.

[7] Di Piazza, M. C., A. Ragusa, and G. Vitale. (2009). Identification of photovoltaic array model parameters by robust linear regression methods. In International Conference on Renewable Energies and Power Quality (ICREPQ'09). 2009.

[8] Khan, S. A., et al. (2021). Chaos Induced Coyote Algorithm (CICA) for Extracting the Parameters in a Single, Double, and Three Diode Model of a Mono-Crystalline, Polycrystalline, and a Thin-Film Solar PV Cell. Electronics, 2021, 10 (17): 2094.

[9] Mdzinarishvili, T., et al. (2020). Determination of the solar rotation parameters via orthogonal polynomials. Advances in Space Research, 2020, 65(7): 1843-1851.

[10] Kabir, F., et al. (2019). Estimation of behind-the-meter solar generation by integrating physical with statistical models. In 2019 IEEE International Conference on Communications, Control, and Computing Technologies for Smart Grids (SmartGridComm). 2019. IEEE.

[11] Mouatasim, A. E. and Y. Darmane. (2018). Regression analysis of a photovoltaic (PV) system in FPO. In AIP Conference Proceedings. 2018. AIP Publishing LLC.

[12] Thomopoulos, N. T. (2012). Essentials of Monte Carlo simulation: Statistical methods for building simulation models. 2012: Springer Science \& Business Media.

[13] Bird, G. (1981). Monte-Carlo simulation in an engineering context. Progress in Astronautics and Aeronautics, 1981, 74: 239-255. 